\title{
Playgol: Learning Programs Through Play
}

\author{
Andrew Cropper \\ University of Oxford \\ andrew.cropper@cs.ox.ac.uk
}

\begin{abstract}
Children learn through play. We introduce the analogous idea of learning programs through play. In this approach, a program induction system (the learner) is given a set of user-supplied build tasks and initial background knowledge (BK). Before solving the build tasks, the learner enters an unsupervised playing stage where it creates its own play tasks to solve, tries to solve them, and saves any solutions (programs) to the BK. After the playing stage is finished, the learner enters the supervised building stage where it tries to solve the build tasks and can reuse solutions learnt whilst playing. The idea is that playing allows the learner to discover reusable general programs on its own which can then help solve the build tasks. We claim that playing can improve learning performance. We show that playing can reduce the textual complexity of target concepts which in turn reduces the sample complexity of a learner. We implement our idea in Playgol, a new inductive logic programming system. We experimentally test our claim on two domains: robot planning and real-world string transformations. Our experimental results suggest that playing can substantially improve learning performance.
\end{abstract}

\section{Introduction}

Children learn through play [Schulz et al., 2007; Sim and Xu, 2017; Sim et al., 2017]. We introduce the analogous idea of learning programs through play. In this approach, a program induction system (the learner) is given a set of user-supplied build tasks and initial background knowledge (BK). Whereas a standard program induction system would immediately try to solve the build tasks, in our approach the learner first enters an unsupervised playing stage. In this stage the learner creates its own play tasks to solve, tries to solve them, and saves any solutions (programs) to the BK. After the playing stage is finished, the learner enters the supervised building stage where it tries to solve the user-supplied build tasks and can reuse solutions learned whilst playing. The idea is that playing allows the learner to discover reusable general programs on its own which can then be reused in the building stage, and thus improve performance. For instance, if trying to learn sorting algorithms, a learner could discover the concepts of partition and append whilst playing which could then help learn quicksort.

To further illustrate our play idea, imagine a child that had never seen Lego before. Suppose you presented the child with Lego bricks and immediately asked them to build a (miniature) house with a pitched roof. The child would probably struggle to build the house without first knowing how to build a stable wall or how to build a pitched roof. Now suppose that before you asked the child to build the house, you first left them alone to play with the Lego. Whilst playing the child may build animals, gardens, ships, or many other seemingly irrelevant things. However, the child is likely to discover reusable and general concepts, such as the concept of a stable wall. As we discuss in Section 2, the cognitive science literature shows that children can better learn complex rules after a period of play rather than solely through observation [Schulz et al., 2007; Sim and Xu, 2017; Sim et al., 2017]. In this paper, we explore whether a program induction system can similarly better learn programs after a period of play.

Our idea of using play to discover useful BK contrasts with most forms of program induction which usually require predefined, often human-engineered, static BK as input [Muggleton et al., 2015; Cropper and Muggleton, 2016a; Law et al., 2014; Schüller and Benz, 2018; Cropper and Muggleton, 2018; Gulwani, 2011; Evans and Grefenstette, 2018; Ellis et al., 2018b]. Our idea is related to program induction approaches that perform multitask or meta learning [Lin et al., 2014; Dechter et al., 2013; Ellis et al., 2018a; Ellis and Gulwani, 2017]. In these approaches, a learner acquires BK in a supervised manner by solving sets of user-provided tasks, each time saving solutions to the BK, which can then be reused to solve other tasks. In contrast to these supervised approaches, our play approach discovers useful BK in an unsupervised manner whilst playing. Playing can therefore be seen as an unsupervised technique for a learner to discover the BK necessary to solve complex tasks, i.e. a form of unsupervised bootstrapping for supervised program induction.

We claim that playing can improve learning performance. To support this claim, we make the following contributions:

- We introduce the idea of learning programs through play and show that playing can reduce the textual complexity of target concepts which in turn reduces the sample complexity of a learner (Section 3). 
- We implement our idea in Playgol, a new inductive logic programming (ILP) system based on meta-interpretive learning (MIL) [Muggleton et al., 2014; Muggleton et al., 2015; Cropper and Muggleton, 2016a] (Section 4).

- We experimentally show on two domains (robot planning and real-world string transformations) that playing can significantly improve learning performance (Section 5).

\section{Related Work}

Program induction approaches learn computer programs from data. Much recent work has focused on task-specific approaches for real-world problems, such as string transformations [Gulwani, 2011]. By contrast, we are interested in general program induction approaches that work on multiple domains. Specifically, we want to develop program induction techniques that discover reusable general concepts, which was the goal of many early AI systems, such as Lenant's AM system [Lenat, 1977].

Program induction approaches use BK as a form of inductive bias [Mitchell, 1997] to restrict the hypothesis space. Most approaches [Muggleton et al., 2015; Cropper and Muggleton, 2016a; Law et al., 2014; Schüller and Benz, 2018; Cropper and Muggleton, 2018; Gulwani, 2011; Evans and Grefenstette, 2018; Ellis et al., 2018b] require as input a fixed, often hand-engineered, BK. To overcome this limitation, several approaches attempt to acquire BK over time [Lin et al., 2014; Dechter et al., 2013; Ellis et al., 2018a; Ellis and Gulwani, 2017], which is a form of meta-learning [Thrun and Pratt, 2012]. In ILP, meta-learning, also known as automatic bias-revision [Dietterich et al., 2008], involves saving learned programs to the BK so that they can be reused to help learn programs for unsolved tasks. Curriculum learning [Bengio et al., 2009] is a similar idea but requires an ordering over the given tasks. By contrast, our approach, and the aforementioned approaches, do not require an ordering over the tasks.

Lin et al. [Lin et al., 2014] use a technique called dependent learning to allow the MIL system Metagol [Cropper and Muggleton, 2016b] to learn string transformations programs over time. Their approach uses predicate invention to reform the bias of the learner where after a solution is learned not only is the target predicate added to the BK but also its constituent invented predicates. The authors show that their dependent learning approach performs substantially better than an independent (single-task) approach. Dechter et al. [Dechter et $a l ., 2013$ ] studied a similar approach for learning functional programs.

These existing approaches perform supervised metalearning, i.e. they need a corpus of user-supplied training tasks. By contrast, our playing approach is unsupervised, where the tasks come not from the user but from the system itself. In other words, our approach allows a learner to discover highly reusable concepts without a user-supplied corpus of training tasks, which Ellis et al. [Ellis et al., 2018a] argue is essential for program induction to become a standard part of the AI toolkit.

Our playing stage is an unsupervised pre-training step. DeepCoder [Balog et al., 2017] also has a pre-training step.
In this step, Deepcoder enumerates every hypothesis in the hypothesis space (up to a depth limit) and generates random input/outputs for each hypothesis. There are many differences between DeepCoder and Playgol. Deepcoder uses the examples and hypotheses to train a neural network to model the distribution over the user-supplied functions in the BK. By contrast, Playgol randomly samples play tasks from the instance space and tries to learn the solutions for them. Whereas DeepCoder learns a distribution over a fixed-set of user-supplied functions, Playgol discovers new programs through play and predicate invention, which can be reused during the building stage. Playgol also uses a dependent learning approach [Lin et al., 2014] to learn a hierarchy of play concepts, where more complex concepts are defined in terms of simpler ones. DeepCoder does not support such abstraction.

Playgol learns a hierarchy of concepts when playing, which can also be seen as an unsupervised approach to discover latent features (i.e. predicates). Dumancic and Blockeel [Dumancic and Blockeel, 2017] use unsupervised pre-training to improve the performance of the ILP system TILDE [Blockeel and Raedt, 1998]. Their CUR ${ }^{2}$ LED approach focuses on learning relational latent representations in an unsupervised manner, and uses clustering to obtain latent features. They show that their approach improves the predictive accuracy of TILDE and reduces the complexity of a learned model (where the complexity refers to the number of nodes in a trained TILDE model). Although Playgol differs from CUR ${ }^{2}$ LED in many ways, both share the goal of discovering new language constructs in an unsupervised manner.

Several studies have shown that children learn better when they have the opportunity to choose what they want to do. Schulz et al. [Schulz et al., 2007] found that children were able to use self-generated evidence to learn about causal systems. Sim and Xu [Sim and Xu, 2017] found that three-year-olds were capable of forming higher-order generalisations about a causal system after a short play period. Sim et al. [Sim et al., 2017] showed that children perform significantly better when learning complex clausal rules through free play or by first engaging in free play and then observing, as opposed to solely through observation. As far as we are aware, there is no research studying whether playing can improve machine learning performance, especially in program induction.

Our idea of learning programs through play is sufficiently general to work with any form of program induction, such as inducing functional programs. However, to clearly explain our theoretical and empirical results, we formalise the problem in an ILP setting using MIL. We use MIL for two key reasons. First, MIL supports learning recursive programs, which is important in the string transformation experiments. Second, MIL uses predicate invention to decompose problems into smaller problems which can then be reused [Cropper and Muggleton, 2016a].

\section{Problem Setting}

We now describe the learning programs through play problem, which, for conciseness, we refer to as the Playgol problem. 


\subsection{Problem Definition}

Given a set of tasks and BK, our problem is to induce a set of programs to solve each task. We formalise the problem in an ILP learning from entailment setting [Raedt, 2008]. We define the input to the problem:

Definition 1 (Playgol input). A Playgol input is a tuple $(\mathcal{H}, \mathcal{E}, B, T)$ where:

- $\mathcal{H}$ is the hypothesis space formed of datalog programs

- $\mathcal{E}$ is the instance space formed of ground atoms

- $B$ is background knowledge represented as a datalog program

- $T$ is set of $k$ build tasks $\left\{E_{1}, E_{2}, \ldots, E_{k}\right\}$ where each $E_{i}$ is a pair $\left(E_{i}^{+}, E_{i}^{-}\right)$where $E_{i}^{+} \subseteq \mathcal{E}$ and $E_{i}^{-} \subseteq \mathcal{E}$ are positive and negative examples respectively of a target predicate represented as ground atoms

Note that in a Playgol input, a build task does not need to have negative examples, i.e. $E_{i}^{-}$may be an empty set.

The Playgol problem is to find a consistent program for each task:

Definition 2 (Playgol problem). Given a Playgol input $(\mathcal{H}, \mathcal{E}, B, T)$, the goal is to return a set of hypotheses $\left\{H_{i} \in\right.$ $\left.\mathcal{H} \mid\left(E_{i}^{+}, E_{i}^{-}\right) \in T,\left(H_{i} \cup B \models E_{i}^{+}\right) \wedge\left(H_{i} \cup B \not \models E_{i}^{-}\right)\right\}$

\subsection{Meta-interpretive Learning}

We solve the Playgol problem using MIL, a form of ILP based on a Prolog meta-interpreter. For brevity, we omit a formal description of MIL, and refer the reader to the literature for more details [Cropper, 2017]. We instead provide an informal overview. A MIL learner is given as input sets of atoms representing positive and negative examples of a target concept, BK in the form of a logic program, and, crucially, a set of second-order formulas called metarules. A MIL learner works by trying to construct a proof of the positive examples. It uses the metarules to guide the proof search. Metarules can therefore be seen as program templates. Figure 1 shows some commonly used metarules. Once a proof is found a MIL learner extracts a logic program from the proof and checks that it is inconsistent with the negative examples. If not, it backtracks to consider alternative proofs.

\begin{tabular}{|l|l|}
\hline Name & Metarule \\
\hline precon & $P(A, B) \leftarrow Q(A), R(A, B)$ \\
postcon & $P(A, B) \leftarrow Q(A, B), R(B)$ \\
chain & $P(A, B) \leftarrow Q(A, C), R(C, B)$ \\
tailrec & $P(A, B) \leftarrow Q(A, C), P(C, B)$ \\
\hline
\end{tabular}

Figure 1: Example metarules. The letters $P, Q$, and $R$ denote secondorder variables. The letters $A, B$, and $C$ denote first-order variables.

\subsection{Sample Complexity}

We claim that playing can improve learning performance. We support this claim by showing that playing can reduce the size of the MIL hypothesis space which in turn reduces sample complexity [Mitchell, 1997] and expected error. In MIL the size of the hypothesis space is a function of the metarules, the number of background predicates, and the maximum program size. We restrict metarules by their body size and literal arity:
Definition 3. A metarule is in $\mathcal{M}_{j}^{i}$ if it has at most $j$ literals in the body and each literal has arity at most $i$.

By restricting the form of metarules we can calculate the size of a MIL hypothesis space:

Proposition 1 (Hypothesis space [Cropper, 2017]). Given $p$ predicate symbols and $m$ metarules in $\mathcal{M}_{j}^{i}$, the number of programs expressible with $n$ clauses is $\left(m p^{j+1}\right)^{n}$.

We use this result to show the MIL sample complexity:

Proposition 2 (Sample complexity [Cropper, 2017]). Given $p$ predicate symbols, $m$ metarules in $\mathcal{M}_{j}^{i}$, and a clause bound $n$, MIL has sample complexity $s$ with error $\epsilon$ and confidence $\delta$ :

$$
s \geq \frac{1}{\epsilon}\left(n \ln (m)+(j+1) n \ln (p)+\ln \frac{1}{\delta}\right)
$$

Proposition 2 helps explain our idea of playing. When playing, a learner creates its own play tasks and saves any solutions to the BK, which increases the number of predicate symbols $p$. The solutions learned whilst playing may in turn help solve the user-supplied build tasks, i.e. could reduce the size $n$ of the target program. To reuse the example from the introduction, if trying to learn sorting algorithms, a learner could discover the concepts of partition and append when playing which could then help learn quicksort. In other words, the key idea of playing is to increase the number of predicate symbols $p$ in order to reduce the size $n$ of the target program. We consider when playing can reduce sample complexity:

Theorem 1 (Playgol improvement). Given $p$ predicate symbols and $m$ metarules in $\mathcal{M}_{j}^{i}$, let $n$ be the minimum numbers of clauses needed to express a target theory with standard MIL. Let $n-k$ be the minimum number of clauses needed to express a target theory with Playgol using an additional $c$ predicate symbols. Let $s$ and $s^{\prime}$ be the bounds on the number of training examples required to achieve error less than $\epsilon$ with probability at least $1-\delta$ with standard MIL and Playgol respectively. Then $s>s^{\prime}$ when:

$$
n \ln (p)>(n-k) \ln (p+c)
$$

Proof. Follows from Proposition 2 and rearranging of terms.

Theorem 1 shows when playing can reduce sample complexity compared to not playing. In such cases, if the number of training examples is fixed for both approaches, the corresponding discrepancy in sample complexity is balanced by an increase in predictive error [Blumer et al., 1989]. In other words, Theorem 1 shows that adding extra (sometimes irrelevant) predicates to BK can improve learning performance so long as some can be reused to learn new programs.

\section{Playgol}

Algorithm 1 shows the Playgol algorithm, which uses Metagol [Cropper and Muggleton, 2016b], a MIL implementation, as the main learning algorithm. Playgol takes as input an instance space (the set of all possible examples) $\mathcal{E}$, initial background knowledge BK, a set of user-supplied build tasks $\mathrm{T}_{b}$, the number of play tasks $p$, and a maximum search depth $\max _{d}$. 
Playgol first enters the unsupervised playing stage. In this stage, Playgol creates its own play tasks $\mathrm{T}_{p}$ by sampling uniformly with replacement $p$ elements from the instance space. We consider this step to be unsupervised because (1) the tasks are not selected by the user, and (2) no labels (i.e. positive or negative) are provided by the user. After creating play tasks, Playgol then uses a dependent learning approach [Lin et al., 2014] to expand the BK. Starting at depth $d=1$, Playgol tries to solve each play task using at most $d$ clauses. To solve an individual task, Playgol calls Metagol. Each time a play task is solved, the solution (program) is added the BK and can be reused to help solve other play tasks. Once Playgol has tried to solve all play tasks at depth $d$, it increases the depth and tries to solve the remaining play tasks. Playgol repeats this process until it reaches the maximum depth $\left(\max _{d}\right)$, then it returns the initial BK augmented with solutions to the play tasks.

Playgol then enters the supervised building stage. In this stage Playgol tries to solve each user-supplied build task using the augmented BK using a standard independent learning approach, eventually returning a set of induced programs.

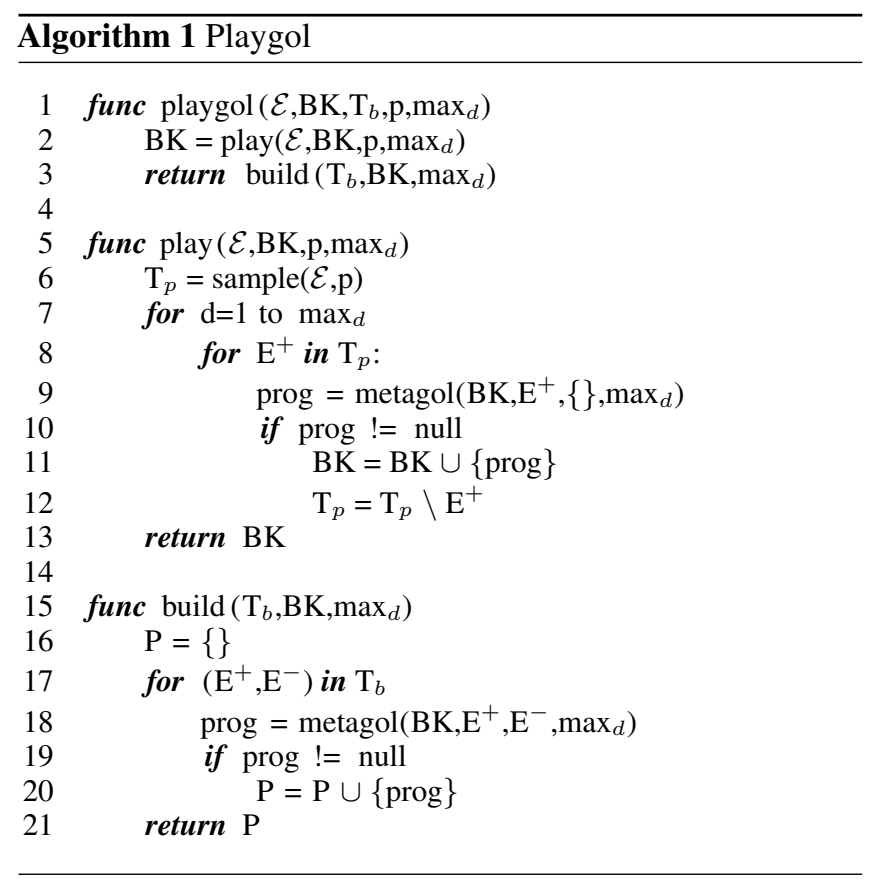

\section{Experiments}

We claim that playing can improve learning performance. We now experimentally test our claim. We test the null hypothesis:

Null hypothesis 1 Playing cannot improve learning performance

Theorem 1 shows that playing can reduce sample complexity compared to not playing. Theorem 1 does not, however, state how many play tasks are needed to improve learning performance. Playgol creates its own play tasks by sampling from the instance space. Suppose we sampled uniformly at random without replacement from a finite instance space. Then if we sample enough times we will sample every instance. One could therefore argue that Playgol is doing nothing more than sampling play tasks that it will eventually have to solve (i.e. Playgol is sampling build tasks whilst playing). To refute this argument we test the null hypothesis:

Null hypothesis 2 Playing cannot improve learning performance without many play tasks

To test null hypotheses 1 and 2 we compare Playgol's performance when varying the number of play tasks. When there are no play tasks Playgol is equivalent to Metagol.

A key motivation for using MIL is that it supports predicate invention. Although we provide no theoretical justification, we claim that predicate invention is useful when playing because it allows for problems to be decomposed into smaller reusable sub-problems. We test this claim with the null hypothesis:

Null hypothesis 3 Saving invented predicates whilst playing cannot improve learning performance

To test null hypothesis 3 we use a variant of Playgol called Playgol $_{N H 3}$. The only difference between Playgol and Playgol $_{N H 3}$ is that Playgol uses all the top-level and invented predicates discovered whilst playing when building. By contrast, Playgol $\mathrm{NH}_{3}$ uses only the top-level predicates discovered whilst playing when building. For instance, suppose that whilst playing both Playgol and Playgol $\mathrm{NH}_{\mathrm{H}}$ discovered quicksort and did so by inventing predicates for the sub-definitions partition and append. Then when building Playgol would use quicksort, partition, and append, whereas Playgol PH3 $_{3}$ would only use quicksort.

\subsection{Robot Planning}

Our first experiment focuses on learning robot plans.

\section{Materials}

There is a robot and a ball in an $n^{2}$ space. The robot can move around and can grab and drop the ball. The goal is to learn a program to move from the initial state to the final state. The robot can perform six dyadic actions to transform the state: up, down, right, left, grab, and drop. Training examples are atoms of the form $f\left(s_{1}, s_{2}\right)$, where $f$ is the target predicate and $s_{1}$ and $s_{2}$ are initial and final states respectively. We allow Playgol to learn programs using the ident and chain metarules (Figure 1). We use $5^{2}$ and $6^{2}$ spaces with instance spaces $X_{5}$ and $X_{6}$ respectively. The instance spaces contain all possible $f\left(s_{1}, s_{2}\right)$ atoms. The cardinalities of $X_{5}$ and $X_{6}$ are approximately $5^{8}$ and $6^{8}$ respectively ${ }^{1}$.

\section{Method}

Our experimental method is as follows. We sample uniformly with replacement 1000 atoms from $X_{n}$ to form the build tasks $T_{b}$. Then for each $p$ in $\{0,200,400, \ldots, 2000\}$, we call $\operatorname{playgol}\left(X_{n}, \mathrm{BK}, T_{b}, p, 5\right)$ which returns a set of programs $P_{p}$. We measure the percentage of correct solutions in $P_{p}$. We enforce a timeout of 60 seconds per play and build task. We measure the standard error of the mean over 10 repetitions.

\footnotetext{
${ }^{1}$ In each state there are $n^{2}$ positions for the robot, $n^{2}$ positions for the ball, and the robot can or cannot be hold the ball, thus there are approximately $2 n^{4}$ states. The instance space contains all possible start/end state pairs, thus approximately $2 n^{8}$ atoms
} 


\section{Results}

Figure 2 shows that Playgol solves more build tasks given more play tasks. For the $5^{2}$ space, Playgol solves only $12 \%$ of the build tasks without playing. The baseline represents the performance of Metagol (i.e. learning without play). By contrast, playing improves performance in all cases. After 1000 play tasks, Playgol solves almost $100 \%$ of the build tasks. For the $6^{2}$ space, the results are similar, where the build performance is only $7 \%$ without playing but over $60 \%$ after 1200 play tasks. These results suggest that we can reject null hypothesis 1 , i.e. we can conclude that playing can improve learning performance.

As already mentioned, one may argue that Playgol is simply sampling build tasks as play tasks. Such duplication may occur. In this experiment, for us to sample all of the build tasks we would expect to sample $\Theta\left(\left|X_{n}\right| \log \left(\left|X_{n}\right|\right)\right)$ play tasks ${ }^{2}$, which corresponds to sampling approximately 5 million and 24 million tasks for the $5^{2}$ and $6^{2}$ spaces respectively. However, our experimental results show that to solve almost all of the build tasks we only need to sample approximately 1000 and 2000 play tasks for the $5^{2}$ and $6^{2}$ spaces respectively. These values are less than $1 / 1000$ of the expected rate. Therefore, our experimental results suggest that we can reject null hypothesis 2 , i.e. we can conclude that playing can improve learning performance without needing to sample many play tasks.

Finally, Figure 2 shows that Playgol solves more tasks than Playgol $_{\mathrm{NH} 3}$, although in the $5^{2}$ space both approaches converge after 2000 play tasks. A McNemar's test on the results of Playgol and Playgol $\mathrm{NH}_{3}$ confirmed the significance at the $p<0.001$ level for the $5^{2}$ and $6^{2}$ spaces. This result suggests that we can reject null hypothesis 3 , i.e. we can conclude that predicate invention can improve learning performance when playing.

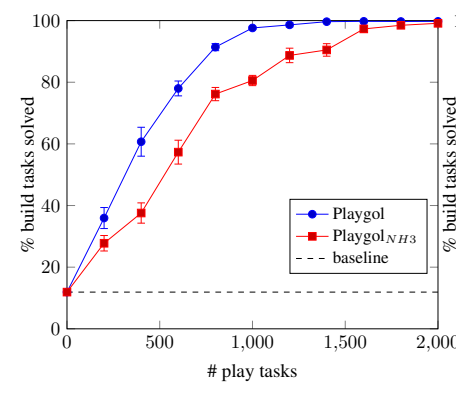

(a) $5^{2}$ space

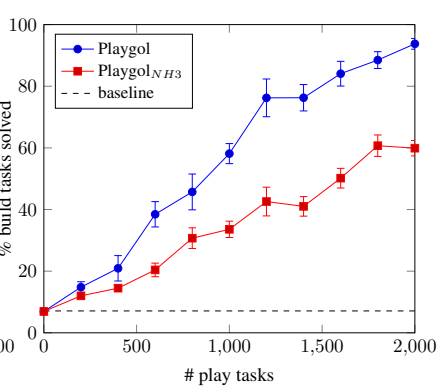

(b) $6^{2}$ space
Figure 2: Robot experiment results. The baseline represents learning without play (i.e. Metagol).

\subsection{String Transformations}

Our first experiment tested the null hypotheses in a controlled experimental setting. We now see whether playing can improve learning performance on 'real-world' string transformations.

\footnotetext{
${ }^{2}$ This problem is an instance of the coupon collectors problem: https://en.wikipedia.org/wiki/Coupon_collector\%27s_problem
}

\section{Materials}

We use 94 real-word string transformation tasks. Our dataset is based on the dataset from [Lin et al., 2014], which in turn is based on [Gulwani, 2011]. We augmented the dataset with manually created tasks, taken from a variety of sources (such as stackoverflow, excel forums, etc). Each task has 10 examples. Each example is an atom of the form $f(x, y)$ where $f$ is the task name and $x$ and $y$ are input and output strings respectively. Figure 3 shows three examples for the build task build_95, where the goal is to learn a program that extracts the first three letters of the month name and makes them uppercase.

\begin{tabular}{l|l} 
Input & Output \\
\hline 22 July,1983 (35 years old) & JUL \\
30 October,1955 (63 years old) & OCT \\
2 November,1954 (64 years old) & NOV
\end{tabular}

Figure 3: Examples for the build_95 string transformation problem.

In the build stage we use the real-word tasks. In the play stage, Playgol samples play tasks from the instance space $X$ formed of random string transformations. The play tasks are formed from an alphabet with 80 symbols, including the letters a-z, A-Z, the numbers $0-9$, and punctuation symbols $(<,>,+,-$ ,,, ,etc). To generate a play task we use the following procedure. We select a random integer $l$ between 3 and 20 to represent the input length. We generate a random string $x$ of length $l$ to represent the input string. We select a random integer $p$ between 3 and 20 and enumerate all programs $P$ of length $p$ consistent with $x$. We select a random program from $P$ and apply it to $x$ to generate the output string $y$ to form the example $f(x, y)$ where $f$ is the play task name. This procedure only generates play tasks for which there is a hypothesis in the hypothesis space. Figure 4 shows example play tasks.

\begin{tabular}{l|l|l} 
Task & Input & Output \\
\hline play_9 & $. f \backslash 73 \backslash R)$ & $\mathrm{F}$ \\
play_52 & $@ \mathrm{~B} 4 \backslash \mathrm{X}_{i} 3 \mathrm{MjKdyZzC}$ & $\mathrm{B}$ \\
play_136 & 9pfy”ktfbS1v & 99PF \\
play_228 & I6zihQk- & $\mathrm{Q}$
\end{tabular}

Figure 4: Example play tasks for the string transformation experiment.

The play instance space $X$ contains all possible string transformations consistent with the aforementioned procedure. The space contains approximately $80^{40}$ atoms ${ }^{3}$.

We provide Playgol with the metarules precon, postcon, chain, and tailrec; the monadic predicates empty, space, letter, number, uppercase, lowercase; the negations of the monadics not_empty, not_space, etc; and the dyadic predicates copy, skip, mk_uppercase, mk_lowercase. These predicates are based on those used in [Lin et al., 2014].

\footnotetext{
${ }^{3}$ In the case that the input is length 20 there are $80^{20}$ possible strings, thus $80^{40}$ pairs.
} 


\section{Method}

Our experimental method is as follows. For each real-word string transformation task $t_{i}$, we sample uniformly without replacement 5 atoms from $t_{i}$ to form the training examples $t_{i, \text { train }}$ and use the remaining 5 atoms as the testing examples $t_{i, t e s t}$. The set of build tasks $T_{b}$ is thus the set of individual tasks, e.g. $T_{b}=\left\{t_{1, \text { train }}, t_{2, \text { train }}, \ldots\right\}$. The set of testing examples $T_{t}$ is likewise $T_{t}=\left\{t_{1, \text { test }}, t_{2, \text { test }}, \ldots\right\}$. For each $p$ in $\{0,200,400, \ldots, 2000\}$, we call playgol $\left(X, \mathrm{BK}, T_{b}, p, 5\right)$ which returns a set of programs $P_{p}$. We measure the predictive accuracy of $P_{p}$ against the testing examples $T_{t}$. We enforce a learning timeout of 60 seconds per play and build task. If Playgol learns no program then every test example is deemed false. We measure the standard error of the mean over 10 repetitions.

\section{Results}

Figure 5 shows the mean predictive accuracies of Playgol when varying the number of play tasks. Note that we are not interested in the absolute predictive accuracies of Playgol, which are low because of the small timeout and the difficulty of the problems. We are instead interested in how the accuracies change given more play tasks, and the difference in accuracies between Playgol and Playgol $\mathrm{NH}_{\mathrm{H}}$. Figure 5 shows that Playgol's predictive accuracy improves given more play tasks. Playgol's accuracy is $25 \%$ without playing. By contrast, playing improves accuracy in all cases. After 2000 play tasks, the accuracy is almost $37 \%$, an improvement of $12 \%$.

Figure 6 shows an example of when playing improved building performance, where the solution to the build task b95 is composed of the solutions to many play tasks. The solutions to the play tasks are themselves are often composed of solutions to other play tasks, including reusing many invented predicates. This example clearly demonstrates the use of predicate invention to discover highly reusable concepts that build on each other.

Overall the results from this experiment add further evidence for rejecting all the null hypotheses.

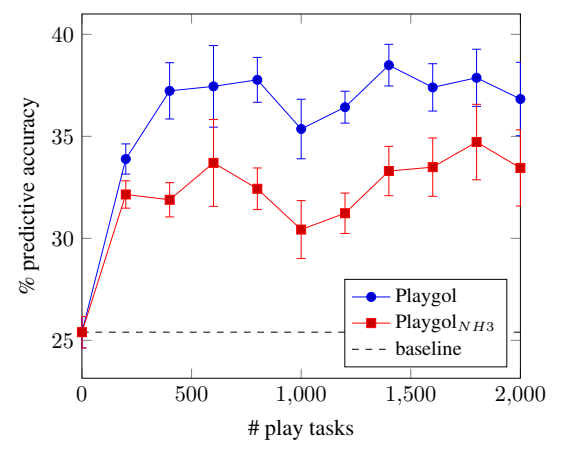

Figure 5: String experiment results.

\section{Conclusions}

We have introduced the idea of learning programs through play. In this approach, a program induction system creates its own play tasks to solve, tries to solve them, and saves any solutions
build_95(A, B):-play_228(A, C), play_136_1(C,B).

play_228(A,B):-play_52(A,B), uppercase(B).

play_228(A,B):-skip(A, C), play_228(C,B).

play_136_1(A,B):-play_9(A,C), mk_uppercase (C,B).

play_9(A,B):-skip (A, C), mk_uppercase $(C, B)$.

play_52(A, B): -skip $(A, C), \operatorname{copy}(C, B)$.

Figure 6: Program learned by Playgol for the build task build_95 (Figure 3). The solution for build_95 reuses the solution to the play task play_228 and the sub-program play_136_1 from the play task play_136, where play_136_1 is invented. The predicate play_228 is a recursive definition that corresponds to the concept of "skip to the first uppercase letter and then copy the letter to the output". The predicate play_228 reuses the solution for another play task play_52. Figure 4 shows these play tasks.

to its BK, which can then be reused to solve the user-supplied build tasks. We claimed that playing can improve learning performance. Our theoretical results support this claim and show that playing can reduce the sample complexity of a learner (Theorem 1). We have implemented our idea in Playgol, a new ILP system. Our experimental results on two domains (robot planning and string transformations) further support our claim and show that playing can substantially improve learning performance without the need for many play tasks. Our experimental results also show that predicate invention can improve learning performance because it allows a learner to discover highly reusable sub-programs.

\subsection{Limitations}

Which domains? Theorem 1 shows conditions for when playing can reduce sample complexity and helps explain our empirical results. Theorem 1 does not, however, help identify the domains where playing is useful. Our preliminary work suggests that playing is useful in other domains, such as to induce graphics programs where playing allows a learner to discover general concepts such as a vertical or horizontal line. Future work should determine the domains where playing is useful.

How many play tasks? Our robot experiments show that as the instance space grows Playgol needs to sample more tasks to achieve high performance. In future work we want to develop a theory that predicts how many play tasks Playgol needs to substantially improve learning performance. In addition, we assume a suitably large instance space. We do not know whether the approach would work when such a space is unavailable.

Better sampling In the string transformation experiment playing did not continue to improve performance as it did in the robot experiment. One explanation for this performance plateau is the relevancy of sampled play tasks. Future work should explore methods to sample more useful play tasks. For instance, rather than create play tasks in an unsupervised manner, it may be beneficial to create play tasks similar to build tasks, i.e. in a semi-supervised manner. 


\section{References}

[Balog et al., 2017] Matej Balog, Alexander L. Gaunt, Marc Brockschmidt, Sebastian Nowozin, and Daniel Tarlow. Deepcoder: Learning to write programs. In ICLR. OpenReview.net, 2017.

[Bengio et al., 2009] Yoshua Bengio, Jérôme Louradour, Ronan Collobert, and Jason Weston. Curriculum learning. In ICML 2019, volume 382, pages 41-48. ACM, 2009.

[Blockeel and Raedt, 1998] Hendrik Blockeel and Luc De Raedt. Top-down induction of first-order logical decision trees. Artif. Intell., 101(1-2):285-297, 1998.

[Blumer et al., 1989] Anselm Blumer, Andrzej Ehrenfeucht, David Haussler, and Manfred K. Warmuth. Learnability and the vapnik-chervonenkis dimension. J. ACM, 36(4):929$965,1989$.

[Cropper and Muggleton, 2016a] Andrew Cropper and Stephen H. Muggleton. Learning higher-order logic programs through abstraction and invention. In IJCAI 2016, pages 1418-1424. IJCAI/AAAI Press, 2016.

[Cropper and Muggleton, 2016b] Andrew Cropper and Stephen H. Muggleton. Metagol system. https://github.com/metagol/metagol, 2016.

[Cropper and Muggleton, 2018] Andrew Cropper and Stephen H. Muggleton. Learning efficient logic programs. Machine Learning, Apr 2018.

[Cropper, 2017] Andrew Cropper. Efficiently learning efficient programs. $\mathrm{PhD}$ thesis, Imperial College London, UK, 2017.

[Dechter et al., 2013] Eyal Dechter, Jonathan Malmaud, Ryan P. Adams, and Joshua B. Tenenbaum. Bootstrap learning via modular concept discovery. In IJCAI 2013, pages 1302-1309. IJCAI/AAAI, 2013.

[Dietterich et al., 2008] Thomas G. Dietterich, Pedro M. Domingos, Lise Getoor, Stephen Muggleton, and Prasad Tadepalli. Structured machine learning: the next ten years. Machine Learning, 73(1):3-23, 2008.

[Dumancic and Blockeel, 2017] Sebastijan Dumancic and Hendrik Blockeel. Clustering-based relational unsupervised representation learning with an explicit distributed representation. In Carles Sierra, editor, IJCAI 2017, pages 1631-1637. ijcai.org, 2017.

[Ellis and Gulwani, 2017] Kevin Ellis and Sumit Gulwani. Learning to learn programs from examples: Going beyond program structure. In IJCAI 2017, pages 1638-1645. ijcai.org, 2017.

[Ellis et al., 2018a] Kevin Ellis, Lucas Morales, Mathias Sablé-Meyer, Armando Solar-Lezama, and Josh Tenenbaum. Learning libraries of subroutines for neurally-guided bayesian program induction. In NeurIPS 2018, pages 78167826, 2018.

[Ellis et al., 2018b] Kevin Ellis, Daniel Ritchie, Armando Solar-Lezama, and Josh Tenenbaum. Learning to infer graphics programs from hand-drawn images. In NeurIPS 2018, pages 6062-6071, 2018.
[Evans and Grefenstette, 2018] Richard Evans and Edward Grefenstette. Learning explanatory rules from noisy data. J. Artif. Intell. Res., 61:1-64, 2018.

[Gulwani, 2011] Sumit Gulwani. Automating string processing in spreadsheets using input-output examples. In $P O P L$ 2011, pages 317-330, 2011.

[Law et al., 2014] Mark Law, Alessandra Russo, and Krysia Broda. Inductive learning of answer set programs. In JELIA 2014, pages 311-325, 2014.

[Lenat, 1977] Douglas B. Lenat. Automated theory formation in mathematics. In Raj Reddy, editor, Proceedings of the 5th International Joint Conference on Artificial Intelligence. Cambridge, MA, USA, August 22-25, 1977, pages 833-842. William Kaufmann, 1977.

[Lin et al., 2014] Dianhuan Lin, Eyal Dechter, Kevin Ellis, Joshua B. Tenenbaum, and Stephen Muggleton. Bias reformulation for one-shot function induction. In ECAI 2014, pages 525-530, 2014.

[Mitchell, 1997] Tom M. Mitchell. Machine learning. McGraw Hill series in computer science. McGraw-Hill, 1997.

[Muggleton et al., 2014] Stephen H. Muggleton, Dianhuan Lin, Niels Pahlavi, and Alireza Tamaddoni-Nezhad. Metainterpretive learning: application to grammatical inference. Machine Learning, 94(1):25-49, 2014.

[Muggleton et al., 2015] Stephen H. Muggleton, Dianhuan Lin, and Alireza Tamaddoni-Nezhad. Meta-interpretive learning of higher-order dyadic datalog: predicate invention revisited. Machine Learning, 100(1):49-73, 2015.

[Raedt, 2008] Luc De Raedt. Logical and relational learning. Cognitive Technologies. Springer, 2008.

[Schüller and Benz, 2018] Peter Schüller and Mishal Benz. Best-effort inductive logic programming via fine-grained cost-based hypothesis generation - the inspire system at the inductive logic programming competition. Machine Learning, 107(7):1141-1169, 2018.

[Schulz et al., 2007] Laura E Schulz, Alison Gopnik, and Clark Glymour. Preschool children learn about causal structure from conditional interventions. Developmental science, 10(3):322-332, 2007.

[Sim and Xu, 2017] Zi L Sim and Fei Xu. Learning higherorder generalizations through free play: Evidence from 2-and 3-year-old children. Developmental psychology, 53(4):642, 2017.

[Sim et al., 2017] Zi Lin Sim, Kuldeep K. Mahal, and Fei Xu. Is play better than direct instruction? learning about causal systems through play. In CogSci 2017, 2017.

[Thrun and Pratt, 2012] Sebastian Thrun and Lorien Pratt. Learning to learn. Springer Science \& Business Media, 2012. 